Revue internationale P.M.E.

Économie et gestion de la petite et moyenne entreprise

Revure

internationale

PME

\title{
Contribution à un modèle pyramidal de la gestion des ressources humaines en PME
}

\section{Mohamed Bayad et Daniel Nebenhaus}

Volume 11, numéro 2-3, 1998

URI : https://id.erudit.org/iderudit/1009048ar

DOI : https://doi.org/10.7202/1009048ar

Aller au sommaire du numéro

Éditeur(s)

Presses de l'Université du Québec

ISSN

0776-5436 (imprimé)

1918-9699 (numérique)

Découvrir la revue

Citer cette note

Bayad, M. \& Nebenhaus, D. (1998). Contribution à un modèle pyramidal de la gestion des ressources humaines en PME. Revue internationale P.M.E., 11(2-3), 161-178. https://doi.org/10.7202/1009048ar
Résumé de l'article

Les développements actuels de la recherche en PME font émerger de nouveaux concepts et modèles propres à éclairer les comportements de gestion des dirigeants de PME. En reprenant certains de ces développements, l'objectif de cet article est de proposer un modèle d'analyse de la PME qui permette une lecture de sa gestion des ressources humaines (GRH). Le dirigeant est le vecteur principal de ce modèle. Par l'impact de sa philosophie de gestion, sa vision de l'organisation et de ses finalités, il est considéré comme la clé de lecture indispensable du système de GRH en PME et, comme tel, il se situe au sommet du modèle pyramidal. Dans le cadre de ce modèle, il revient au dirigeant de construire son propre système de relations avec ses ressources humaines. 


\title{
Contribution à un modèle pyramidal de la gestion des ressources humaines en PME
}

\author{
Mohamed BAYAD \\ CESREM, Université de Metz \\ Daniel NEBENHAUS ${ }^{1}$ \\ LAMSADE, Université Paris IX-Dauphine
}

\section{MOTS CLÉS}

Dirigeant - PME - GRH - Organisation - Théorie - Modèle - Vision

\begin{abstract}
RÉSUMÉ
Les développements actuels de la recherche en PME font émerger de nouveaux concepts et modèles propres à éclairer les comportements de gestion des dirigeants de PME. En reprenant certains de ces développements, l'objectif de cet article est de proposer un modèle d'analyse de la PME qui permette une lecture de sa gestion des ressources humaines (GRH). Le dirigeant est le vecteur principal de ce modèle. Par l'impact de sa philosophie de gestion, sa vision de l'organisation et de ses finalités, il est considéré comme la clé de lecture indispensable du système de GRH en PME et, comme tel, il se situe au sommet du modèle pyramidal. Dans le cadre de ce modèle, il revient au dirigeant de construire son propre système de relations avec ses ressources humaines.
\end{abstract}

\begin{abstract}
Contemporary research on SMB enlight the birth of new concepts and models about the managerial behavior of their CEO. Retrieving some of these developments, this article aims to propose an analytical and contigential model of the Small and Medium Business Enterprises, which offers a reading of their HRM practices. The CEO is the main vector of the model. The impact of his managerial philosophy, his vision of the firm and its goals, make the authors consider he is the key of the HRM system in a SME. For that reason, he is located at the top of the pyramidal model developped herein, and it depends on him to build his own relationships with the personnel.
\end{abstract}

1. En séjour sabbatique au Babson College en 1997-1998 avec une bourse de la FNEGE. 


\section{RESUMEN}

Los avances actuales en la investigación dentro del ámbito de las PyMEs han favoricido la emergencia de nuevos conceptos que nos permeten entender mejor las funciones y actividades de gestión características de los directivos y gestores de las PyMEs. A partir de algunos de esos avances, el objetivo de este artículo es proponer un modelo de análisis de la PyME que nos permita una interpretación de su gestión de recursos humanos (GRH). El directivo es el vector principal que sive de soporte a este modelo. En función del impacto provocado por su filosofia de gestión, así como por su percepción especifica de la organización y de sus finalidades, éste debe ser considerado como el factor clave para poder entender el sistema del GRH en las PyMEs dado que se halla situado en el vértice de un modelo de tipo piramidal. En el marco de este modelo, podemos estimar que la capacidad para poder configurar su propio sistema de relaciones, a partir de sus recursos humanos disponibles, es algo que deriva directamente del nivel de competencia del gestor directivo.

\section{Introduction}

Les recherches en gestion s'inscrivent obligatoirement dans un cadre conceptuel modélisé qui permet ensuite de tester, en vue de leur validation, des hypothèses de travail. Le travail de recherche se poursuit alors en mettant en œuvre une méthodologie (quantitative ou qualitative ou les deux à la fois) appropriée à un terrain et à une problématique. C'est par la mise en relation de variables et de concepts que se construit un modèle de recherche, plus ou moins formalisé, qui sera ensuite validé au cours d'une phase dite d'opérationalisation.

À chacun des niveaux de ce processus de recherche se pose le problème de la délimitation des disciplines en sciences de gestion : à la nécessaire autonomie plus ou moins relative de chaque domaine (finances, marketing, ressources humaines, etc.) s'oppose l'impératif de transversalité. Ainsi, la définition de l'objet même de la recherche et de la claire délimitation de son champ prennent une plus grande acuité. Cette préoccupation s'impose, cependant, de manière de plus en plus visible dans

\section{LES AUTEURS}

Mohamed Bayad est maître de conférences en sciences de gestion à l'ESM - Université de Metz. II est aussi responsable du DESS « Entrepreneuriat et développement des PME » ainsi que de l'équipe de recherche "Ressources humaines et organisation » du CEREMO. Ses thèmes de recherche sont les suivants : interface entre management stratégique et GRH en contexte entrepreneurial/PME et interculture.

Adresse: 3, Place Fdouard-Branly 57070 Metz, France. Courriel : bayad@esm.univ-metz.fr

Daniel Nebenhaus est un ancien élève de l'École des HEC (Jouy-en-Josas), titulaire d'un doctorat en sciences de gestion et maître de conférences à l'Université de Paris-Dauphine. II vient de passer une année sabbatique au Babson College (États-Unis) comme "visiting research scholar ». Depuis dix ans, ses travaux portent sur la GRH en PME. II est auteur de plusieurs articles sur le sujet. 
le champ des petites et moyennes entreprises (PME), et les problématiques reposent sur une combinaison des démarches quantitatives et qualitatives.

Les travaux de recherche sur les PME mettent fréquemment en évidence une difficulté théorique contingente à ce domaine : les limites ou, le plus souvent, l'inadéquation des modèles de gestion reconnus au niveau de la grande entreprise (GE). Dans les représentations utilisées pour la GE, les spécificités rencontrées dans l'étude des PME ne sont pas prises en compte. Au-delà même de la variété intrinsèque de la notion de PME, sur laquelle il serait bon de revenir ultérieurement, plusieurs auteurs dressent un constat similaire : les représentations théoriquement valables pour la $\mathrm{GE}$ ne permettent pas de comprendre le fonctionnement réel de la PME, ou sont même parfois structurellement inadaptées ${ }^{2}$ (Johannisson, 1994 ; Belletante, 1991 ; Julien et Marchesnay, 1988 ; D’Amboise, 1982).

La première partie de cet article montre qu'il existe d'ores et déjà plusieurs modélisations théoriques concernant soit la PME dans sa globalité, soit des domaines de gestion qui s'y appliquent. Presque sans exception, les concepts mis en œuvre font état d'une double particularité qu'il faut saisir pour se représenter la gestion de la PME et l'expliquer correctement : le rôle du dirigeant avec son statut (propriétaire ou non) et l'importance des relations de l'entreprise avec son environnement, lesquelles se présentent souvent sans lien apparent immédiat avec l'activité ou parfois sous la forme de réseau.

La seconde partie sera consacrée à l'ébauche d'un modèle de gestion des ressources humaines $(G R H)$ de la PME. Cette démarche ne vise pas à proposer une représentation théorique universelle pour les PME, mais plus simplement, à intégrer au domaine de la GRH les apports et les invariants constatés lors de l'étude d'autres modèles théoriques et concepts appliqués à la PME.

Les chercheurs en gestion de la PME ont également comme point commun de souligner une seconde catégorie de difficultés qu'ils estiment propres à leur domaine : celles concernant les méthodologies d'observation et de recueil de l'information. Par exemple, pratiquement tous rejettent la collecte des données à partir de l'envoi d'un questionnaire postal. L'importance de la connaissance, ne serait-ce que fugitive, de l'entreprise, à travers la passation d'un questionnaire «en direct » pendant deux ou trois heures est primordiale pour la fiabilité de la collecte des données. Dans les PME, savoir qui répond, et dans quelles conditions, est aussi important que le contenu des réponses. Par ailleurs, la qualité de l'information

2. En effet, quels rapports entre la très petite entreprise (TPE) artisanale ou de commerce au sein de laquelle travaille une, deux ou trois personnes, et la moyenne entreprise (ME) qui emploie plusieurs centaines de salariés et dont la structure est comparable à celle d'une GE ? Cette question de la variabilité des approches et des comportements en fonction de la taille selon une typologie admise (TPE, PE, ME, MGE) serait à réexaminer à la lumière d'un modèle d'analyse de la GRH en PME, qui est d'ores et déjà opératoire. 
dépend également de l'intensité de l'échange qui aura lieu avec l'interlocuteur interviewé, haut responsable ou dirigeant de la PME.

\section{Quelques modèles théoriques propres aux PME existent déjà ou sont en cours de validation}

Trouver, définir précisément et mettre en œuvre un modèle ad hoc de représentation de la PME connaît d'ores et déjà des débuts de réponse satisfaisante dans plusieurs domaines de recherche. Illustrons ce propos à l'aide de plusieurs exemples.

\subsection{La PME, le dirigeant, le propriétaire dirigeant et les PMO}

Précisons en premier lieu les concepts de dirigeant et de propriétaire-dirigeant : ces deux expressions permettent de distinguer les travaux sur la PME de ceux qui ont comme objet des PMO, c'est-à-dire tout type de petite structure, éventuellement filiale de grands groupes. Dans ce dernier cas, le statut du dirigeant n'est ni celui d'un propriétaire, ni celui d'un responsable choisi par les propriétaires et en rapport direct avec eux. C'est un cadre supérieur qui dirige une filiale ou un département, et ses responsabilités s'inscrivent dans un déroulement de carrière. Dans ce cas de figure, les problèmes de gestion abordés relèvent principalement des GE et se posent en termes de décentralisation et de contrôle, de différenciation et d'intégration, etc.

Les travaux dans le champ des PME doivent donc au préalable définir le type d'entreprise qui sera ainsi caractérisé. Alors que les statistiques publiques et les chercheurs ne retiennent que le critère «effectifs » (moins de 500 salariés), la loi et la réglementation française dans le domaine social, le droit des sociétés ou la fiscalité, énoncent fréquemment deux critères : les effectifs et le chiffre d'affaires. L'effet de seuil jouera sur l'un ou l'autre de ces critères. Dans d'autres pays, où il existe une définition officielle de la PME, on rencontre des exemples d'utilisation de ces deux critères (voir D'Amboise et Garand, 1993).

Pour caractériser le champ des PME, la première variable est la taille de l'entreprise. Mais, comme on l'a vu plus haut, cela ne suffit pas, car une petite ou moyenne organisation n'est pas toujours une PME. Le statut du dirigeant confère une caractéristique ontologique de la PME : elle possède la propriété fondamentale d'être un centre de décision autonome. Autonome voulant dire que le dirigeant de la structure étudiée joue un rôle crucial dans tout processus décisionnel important pour l'entreprise. En aucune manière, son autorité ne saurait dépendre d'une instance relevant d'un groupe ou d'une maison mère. Dans le cas de figure où le dirigeant n'est pas simultanément propriétaire de l'entreprise, nous émettons l'hypothèse que les relations avec l'actionnariat sont d'une autre nature que celles du dirigeant de filiale avec sa maison mère. Une tentative de transposition de la théorie du mandat pourrait peut-être servir à éclairer la vraie nature de ces relations. 


\subsection{La vision entrepreneuriale comme paradigme de la stratégie de la PME}

En stratégie, le concept de vision entrepreneuriale (D’Amboise et Bouchard, 1990 ; Cossette, 1993) apparaît comme prometteur, car sa capacité explicative est extrêmement élevée. La puissance d'un outil, tel que la carte cognitive (Cossette, 1993) utilisée pour comprendre comment et dans quelle direction le propriétaire dirigeant projette l'entreprise, est certainement insuffisamment exploité si on le cantonne à la stratégie. Les domaines connexes en PME, à savoir l'organisation et la GRH, devraient voir s'appliquer avec profit ce type de méthode de recherche.

L'apport du concept de vision pour l'étude des PME permet le développement d'une voie de recherche intéressante. Comme l'ont rappelé D'Amboise et Bouchard (1990) dans leur recension de la documentation sur le sujet, les premiers travaux et publications datent tout au plus d'une quinzaine d'années. Ils distinguent quatre groupes thématiques, soit le leadership, la culture organisationnelle, la stratégie et le contexte entrepreneurial. Ils notent qu'il peut exister des liens entre la vision, la stratégie et la performance d'une organisation, aussi bien en milieu PME que dans la grande firme, sans qu'ils aient été théoriquement démontrés. Enfin, ils remarquent, et ceci est tout à fait intéressant pour notre propos, que la vision pourrait influencer la réussite d'une organisation sur le plan des ressources humaines et économiques.

Par la suite, le travail de Filion (1991) et les développements appliqués de Cossette (1993) dans l'utilisation de la cartographie cognitive comme technique de mise au jour de la vision du dirigeant d'entreprise insistent sur une dimension essentielle, mais non exclusive : la vision stratégique. À notre avis, cette perspective est réductrice, eu égard au potentiel offert par le concept de vision. Même si Filion définit la vision par rapport à l'avenir de l'entreprise, à la stratégie (le couple produitmarché) et au type d'organisation, la dimension qui importe le plus dans cette définition est bel et bien la stratégie. Sur ce point, la définition de D'Amboise et Bouchard, pour qui la vision du dirigeant d'entreprise c'est l'état futur et possible de l'organisation, est potentiellement plus riche à cause, peut-être, de son caractère plus général.

\subsection{Autour de la théorie des réseaux : de nouveaux concepts pour la PME}

En économie industrielle et en économie d'entreprise, la théorie des réseaux et le cas, plus particulier, des districts industriels ont donné naissance à toute une école de pensée formée à son origine d'économistes et de sociologues de formation (Piore et Sabel 1984). Aujourd'hui, un mouvement de recherches en gestion, trop peu connu en France, s'inscrit dans cette optique en utilisant le paradigme du réseau pour améliorer les connaissances relatives aux comportements des PME avec leurs 
facteurs de succès et d'échecs (croissance, exportation, stratégie, gestion de la maind'œuvre, etc.).

En matière de réseaux, pour être plus précis, distinguons deux catégories de réseaux d'entreprises dans lesquels s'insèrent les PME. Il s'agit, d'une part, et principalement de réseaux constitués par un ensemble d'entreprises de petite ou moyenne taille qui sont installées dans un espace géographique limité. La communauté locale, la vallée, une petite région sont les archétypes de regroupements les plus classiques. Lorsque ce type de réseau repose sur le maintien d'une tradition industrielle préservée et accentuée par la présence d'une main-d'œuvre qualifiée, on aura affaire à une activité de type mono-industrie. On parle alors de district industriel, où le processus de production peut faire peu ou pas appel aux technologies de pointe. Le parc technologique apparaît comme une autre catégorie, dont un critère de regroupement est géographique, mais qui, parfois, se caractérise par des activités souvent variées d'une PME à l'autre, mais de haut niveau et d'innovation technologique. Le Parc technologique bio-industriel donne un exemple spécialisé de la catégorie précédente.

D'autre part, il existe aussi un réseau organisé quand un grand groupe industriel développe un savoir-faire relationnel avec un ensemble de dizaines, voire de centaines, de sous-traitants et de fournisseurs. Dans ce réseau, on peut trouver aussi bien des PME que des grandes entreprises. L'application du concept de réseau dans ce cadre déterminé ne fournit pas, en l'occurrence, une grille pertinente pour comprendre ou expliquer les comportements des PME. En effet, la grande entreprise à la tête du réseau joue le rôle d'acteur moteur à l'origine de la stratégie dans ce cas. Les autres partenaires doivent suivre et s'adapter autant que faire se peut, s'ils désirent poursuivre leur coopération avec le client-donneur d'ordres et ne pas se priver de ce marché.

L'approche proposée par Johannisson (1994) pour analyser le processus d'internationalisation des PME fournit un excellent exemple pour illustrer à nouveau notre propos. D'une part, ce chercheur confirme qu'en matière de gestion de la PME il s'avère utile de reconsidérer les catégories habituelles de pensée. Il remet en cause les freins habituellement retenus au développement à l'exportation : le manque simultané de motivation et de compétences requises. Il conteste également le processus « linéaire » d'élargissement de la taille du marché : du local au régional, puis au national avant de s'intéresser à l'international. À l'opposé, il montre que par un processus qu'il qualifie du néologisme de «glocal», les PME peuvent passer d'emblée d'un marché géographiquement limité à l'internationalisation. D'autre part, pour sa démonstration, il s'appuie sur l'analyse en termes de réseau (district industriel ou parc technologique) qu'il enrichit cependant de relations sociales résiliaires. Les relations de cette nature, extrêmement sophistiquées, apparaissent aussi bien comme des relations d'affaire d'entreprise à entreprise que des rapports sociaux entre dirigeants : liens familiaux, de voisinage, etc. L'auteur 
souligne par ailleurs que les logiques sont différentes selon le type de réseau: district industriel ou parc technologique.

La stratégie de marché qu'il qualifie de « glocal» résulte de la combinaison d'opérations commerciales globales et de coopérations locales entre PME, d'où le néologisme qui résulte de la contraction des deux termes. Dans cette analyse se confirment la place et le rôle central de l'entrepreneur propriétaire-dirigeant: de sa capacité à maîtriser et à combiner les deux niveaux de relation résulte le potentiel de son entreprise à pouvoir accéder à un marché étranger.

\subsection{L'entrepreneuriat ou le dirigeant au poste de commande}

L'entrepreneuriat a acquis une reconnaissance en tant que domaine de recherche en gestion, en se structurant sur le plan international à travers l'existence de revues, de congrès et d'équipes. Ce courant développe une approche de la PME et de son développement, exclusivement autour de la question du chef d'entreprise. Bien que ce champ soit encore très peu développé en France, il n'en constitue pas moins un domaine fertile, et ses conséquences pratiques en termes de politiques de soutien à la création d'emplois sont reconnues.

Traditionnellement, le principal facteur reconnu de motivation de l'entrepreneur est l'indépendance (Brockaus, 1982 ; Vesper, 1980), même si de plus en plus d'auteurs insistent sur l'importance de la variable relationnelle dans la réussite de l'entreprise (Shapero et Sokol, 1982).

Une autre approche de la vision entrepreneuriale, radicalement différente des précédentes, est celle développée par Julien et Marchesnay (1988) qui, pour analyser les stratégies des PME, proposent une typologie simple en deux classes de profils du dirigeant : l'entrepreneur PIC ou CAP. Ils expliquent aussi que le comportement type adopté par chaque entrepreneur dépend de ses caractéristiques : milieu, éducation, psychologie et données contingentes de l'environnement de l'entreprise. Ici encore, des auteurs insistent sur l'importance à accorder au dirigeant et à l'environnement. Par ailleurs, les auteurs (Bayad et Nebenhaus, 1993) avaient montré l'existence de liens entre le profil du dirigeant et ses préoccupations dans le domaine de la GRH.

Un courant de pensée (Lorrain et Dussault, 1988 ; Gartner, 1989) prend appui sur les études de Mintzberg et Waters (1985) pour développer une recherche sur les activités au quotidien du dirigeant de PME plutôt que sur son profil personnel. La classification des activités obtenues, à partir d'une synthèse de la littérature, permet à Lorrain, Belley et Ramagalahy (1994, p. 14) de proposer un modèle selon lequel un dirigeant assume trois groupes d'activités.

La première dimension réfère à des activités de gestion, dans lesquelles le dirigeant prévoit l'avenir de son entreprise (la planification); il élabore la structure d'organisation (l'organisation); mobilise les ressources humaines (la direction); et effectue le suivi 
des réalisations et procède aux ajustements (contrôle). Dans la deuxième dimension, qui est de nature fonctionnelle, le dirigeant assume des activités de nature administrative (l'administration); il développe des relations externes et assure la promotion des intérêts de son entreprise (les relations publiques) [...] ; il résout des problèmes ou des crises liés aux activités quotidiennes (la régulation). Enfin, dans la troisième dimension, de nature opérationnelle, le dirigeant (exécutant) assure l'approvisionnement $[\ldots]$, la maintenance $[\ldots]$ et $[\ldots]$ la vente.

\subsection{Territoire, réseaux et relations appliqués à la stratégie financière}

En finance d'entreprise, il existe également quelques tentatives de développement d'une approche théorique et conceptuelle nouvelle, tel le concept de territoire financier élaboré par Belletante (1991). L'auteur s'intéresse à la double question des décisions et de la stratégie financière des PME. Pour cela, il a besoin d'un outil d'analyse théoriquement justifié permettant d'appréhender globalement la finance des PME. Le cadre théorique habituel, issu de l'analyse néoclassique et construit indépendamment de la taille des entreprises, ne permet en effet pas de rendre compte correctement du comportement financier des PME.

L'auteur se demande comment satisfaire le besoin de financement des PME dans un contexte où l'appel au financement par le marché se révèle impraticable, compte tenu de la taille de ces entreprises. Par conséquent, un modèle reposant sur les informations communiquées au marché n'apparaît pas justifié sur le plan théorique. Ainsi, pour analyser les comportements en termes de décision et de stratégie, il semble utile de faire appel à de nouveaux concepts; et l'auteur propose celui de territoire financier, adapté aux spécificités de la gestion des PME. Le territoire financier vise alors à tenir compte du caractère hétérogène de l'accès aux capitaux. Ce caractère hétérogène dépend en fait de deux facteurs :

- savoir si c'est un cas de relation de type actionnaires-dirigeants, avec les risques de conflit que cela implique, ou dans un cas de propriétairedirigeant qui bénéficie d'une information asymétrique au regard des autres apporteurs de capitaux?

- connaître le traitement dont bénéficie l'entreprise de la part de son banquier, et en particulier le type d'instrument financier qui lui sera proposé.

Font donc partie du territoire financier le centre de décision, c'est-à-dire les acteurs qui participent à la décision financière, les partenaires, qui apportent aussi bien des capitaux que des services, et les produits.

L'intérêt de cette approche repose sur deux idées obligatoires dans tout travail théorique sur les PME : le rôle du dirigeant (avec la distinction propriétaire ou non) et l'importance des relations de la firme à son environnement. L'auteur ne mentionne néanmoins aucune référence au concept de réseau. 


\section{De la place stratégique de la GRH en PME, à la définition d'un modèle}

\subsection{Quel type de modèle de GRH de la PME ?}

La première partie de cette étude a consisté à montrer que les modèles explicatifs $\mathrm{du}$ fonctionnement des GE et de leurs processus décisionnels ne pouvaient pas s'appliquer ou être transférés directement au champ des PME. Les nouvelles approches dans la recherche sur les PME émergent de chercheurs qui ont su acquérir une connaissance du fonctionnement réel de ces entreprises. Fort logiquement, chacun de leur côté, ils arrivent à la conclusion que les pratiques existent, mais ne se présentent pas sous la forme connue en GE.

Vraisemblablement, une des raisons principales de cet état de fait tient à l'hétérogénéité du champ considéré. En effet, contrairement aux GE, pour les PME la difficulté tient à mettre en évidence des invariants de gestion : que ce soit par l'usage d'outils, de processus décisionnels ou, a fortiori, par l'appréhension des pratiques qui se distinguent par leur faible formalisation administrative. Les particularités de la PME énoncées par Scott (voir D’ Amboise et Garand, 1993), à savoir « une taille organisationnelle réduite, une forte centralisation autour du propriétairedirigeant, une vision entrepreneuriale commune, un très faible degré de formalisation des activités de gestion et de production, une flexibilité accrue face aux changements, et une implacable vulnérabilité face à la concurrence à tous les niveaux », fournissent en effet les clés permettant de comprendre les raisons de cette absence d'homogénéité du milieu PME.

Par ailleurs, la formalisation théorique de ce champ d'étude peut être envisagée globalement ou focalisée sur un domaine de gestion : finance, stratégie, marketing, innovation... ou GRH! Quand le choix porte sur une des branches des sciences de gestion, la recherche des signes de compatibilité entre le modèle spécifique et un modèle global permet de conforter simultanément les deux familles de concepts. Par voie de conséquence, le modèle spécifique apporte des éléments de confirmation au modèle général et trouve en partie des justifications à sa propre existence. Néanmoins, même si le découpage fonctionnel facilite la démarche méthodologique du chercheur, il va souvent à l'encontre du mode d'organisation entrepreneuriale qui sollicite par nature une lecture transversale du fonctionnement réel de la petite entreprise.

À travers l'étude de la dimension sociale de la gestion, deux grandes tendances de travaux sur la GRH en milieu de PME, mais aussi sur les PME, en général ressortent : la transposition et l'adaptation d'approches GE en contexte de PME; et dans ce cas, inévitablement, le raisonnement évoque les manques, les lacunes, l'absence totale ou partielle de pratiques. Dans ce contexte, l'existence d'outils de gestion repose implicitement ou non sur leur formalisation. À l'opposé, des tentatives 
récentes tirent des conclusions des pratiques observées et tentent de jeter un regard neuf sur la question.

Les propositions de concepts et de modèles spécifiques ont deux points communs :

- la place accordée au rôle et au statut du dirigeant;

- la fonction dévolue aux relations de l'entreprise à son environnement.

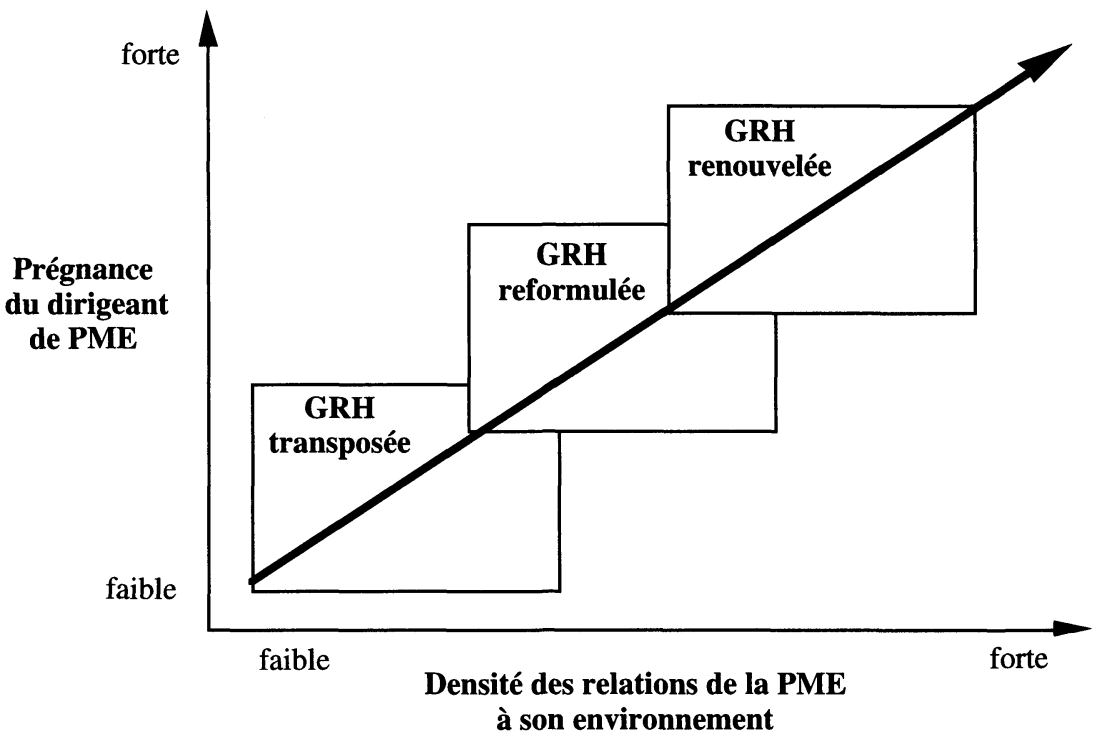

Partant de cette typologie, la transposition et l'adaptation ou l'innovation, l'esquisse d'un modèle de recherche se rattachant au second type est avancée dans cet article. Il se confirme en effet que les démarches visant à vérifier si les PME utilisent ou non des outils de gestion créés par et pour la GE ne permettent ni de mesurer le degré de sophistication ou l'intensité et la qualité des pratiques de gestion des PME, ni de comprendre le caractère global des intentions (la volonté) du propriétaire-dirigeant et des processus de gestion qui y sont mis en œuvre (les activités, les personnes et le contexte organisationnel). Ce dernier point apparaît d'ailleurs à la fois comme essentiel à la compréhension de la façon dont sont pensées (mission), projetées (vision stratégique) et animées (philosophie de gestion) les PME par leurs propriétaires-dirigeants. Il prend place, par conséquent, au cœur de tout modèle théorique pertinent de la PME.

La pertinence du concept clé de vision stratégique de l'entrepreneur conduit à proposer en premier lieu son adaptation à la GRH. Ensuite, le modèle proposé suggère une manière appropriée d'appliquer conceptuellement aux PME la réalité d'un modèle contingent tripolaire : stratégie - organisation/structure - GRH. 


\subsection{Proposition d'un modèle de base de la GRH de la PME}

\subsubsection{Le modèle de base de la PME}

Dans son étude pour le compte de l'OCDE sur la technologie et les PME, Julien (1993) énumère les cinq variables clés dégagées sur une base empirique, qui fondent leur compétitivité. Parmi ces variables, il énumère dans l'ordre: le rôle du dirigeant (propriétaire ou non), l'organisation et la flexibilité. Les deux dernières variables relèvent plus spécifiquement de la technologie. Sur une base théorique, Marchesnay (1997) met en exergue les spécificités de la démarche stratégique de la petite entreprise. Base de la compétitivité, ces spécificités donnent lieu à une grille d'analyse permettant de faire ressortir les variables clés du système stratégique de la PME.

En fait, celles-ci recouvrent les divers ordres de préoccupations dans la recherche en stratégie des PME et surtout des PE : tout d'abord, les buts des dirigeants, liés à leur profil entre autres ; ensuite, l'organisation de l'entreprise, comprenant toutes sortes de configurations, au sens de Mintzberg et Waters (1985); les activités, leur nature et leur nombre ; enfin, l'environnement, ou plutôt les types d'environnements qui concernent la stratégie de la petite entreprise.

Le modèle de recherche proposé pour l'analyse de la GRH en PME réduit le nombre de variables clés présentées par Marchesnay (1991) et Julien (1993) et les ordonnent différemment. Ce modèle, encore à l'état d'esquisse, fait référence à l'idée de pyramide. La forme d'une pyramide, objet mathématique connu depuis la plus haute antiquité, dépend en réalité de deux éléments d'égale importance:

- sa base, dessinée comme un carré, ou un triangle équilatéral ;

- la hauteur de son sommet.

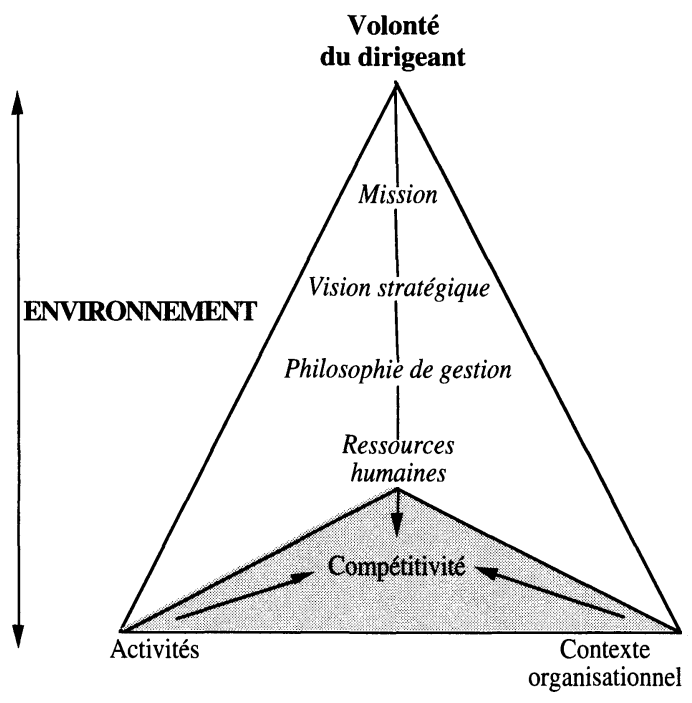


Inspirée du modèle entrepreneurial de KAO (1991), la base pour ce modèle est de type triangulaire. Elle met en interrelation trois variables clés :

- les ressources humaines; il s' agit des hommes et des femmes qui besognent dans les PME avec leurs qualités, leurs compétences, leurs motivations et leurs attentes. Comme l'ont déjà montré différents travaux de recherche (D'Amboise, 1991 ; D'Amboise et Garand, 1993 ; Bayad et Nebenhaus, 1993), les ressources humaines constituent un facteur décisif de succès (ou d'échec) des PME, des sources de difficultés ou, au contraire, un avantage compétitif majeur;

- les activités ; elles correspondent à ce qu'un groupe de personnes ou l'organisation dans son ensemble doit réaliser afin de remplir la mission de l'entreprise. Les activités transparaissent au niveau des principales fonctions de gestion. Elles relèvent ainsi, tout autant des choix organisationnels et des personnes qui en ont la charge, que de l'influence de l'environnement externe et de la manière d'y répondre (stratégies marketing, financières, technologiques, etc.);

- le contexte organisationnel : il comprend la structure formelle ou informelle de l'organisation, les systèmes de gestion, les rôles des acteurs, la culture comme résultante de l'interface des valeurs du dirigeant et de celles des membres de l'organisation.

Ces trois variables de base dépendent de deux spécificités fortes de la PME : le dirigeant (sommet de la pyramide ${ }^{3}$ ) et l'environnement externe.

Concernant le dirigeant, le milieu des consultants travaillant pour les PME et en particulier ceux qui œuvrent aux rapprochements, à la transmission ou à la reprise admet une opinion non issue de travaux de recherche : «le départ d'un dirigeant revient à couper la tête de l'entreprise ». C'est donc dire à quel point la gestion et la stratégie sont fortement dépendantes de sa personnalité. Ainsi, et par voie de conséquence, la performance et l'absence de pratiques formelles ne sauraient égaler en valeur une absence totale de pratiques.

L'institutionnalisation plus marquée dans la GE de pratiques et d'outils de gestion formalisés conduit à pérenniser le fonctionnement de l'organisation sur une certaine base, quelle que soit l'efficience réelle de ce fonctionnement. Inversement, dans les PME, qui par nature ont moins d'outils de gestion formalisés, la dépendance à l'égard du dirigeant s'accroît en proportion inverse. C'est ce dernier qui souvent met en œuvre des pratiques informelles qui n'en sont pas moins efficientes.

3. Mathématiquement, le sommet de la pyramide est un point de jonction unique où tous les paramètres de la base se rejoignent obligatoirement. 
Cette forte personnalisation rejaillit donc sur le degré de formalisation, le poids des activités jugées stratégiques et le choix des personnes. Le terme de volonté (sommet de la pyramide) apparaît alors comme le plus approprié et synthétique pour appréhender cette personnalisation de la PME. L'étendue de cette personnalisation (cœur de la pyramide), reflet des buts et des objectifs du dirigeant, s'approche à travers la mission (Qui sommes-nous ? Quel est notre métier? Qu'est-ce qui justifie notre existence en tant que structure indépendante ? Comment pouvons-nous nous définir et en quoi sommes-nous différents des autres entreprises ? En somme, quelle est notre légitimité sociale ?), la vision stratégique (D'où venons-nous ? Où voulonsnous aller et comment? En somme, quel avenir voulons-nous ?) et la philosophie de gestion (Comment entend-on répondre aux aspirations des employés? Quelle organisation du travail choisir? Quelles activités privilégiées? Quelles relations avoir avec les clients, les fournisseurs, les syndicats, l'État et les collectivités, etc. ? En somme, quel est le style de management?).

L'environnement détermine tout d'abord le côté durable ou non des avantages compétitifs de la PME, face à la concurrence et aux mutations technologiques sur des créneaux de marché étroits et friables. Source de menaces, l'environnement est aussi une source d'occasions d'affaires par les ressources externes disponibles (matérielles et immatérielles) que la PME transforme en capacités et en compétences propres et distinctives. Il convient aussi de préciser, avec Marchesnay (1997), «les relations qui s'établissent entre l'organisation interne et l'organisation externe, c'est-à-dire les réseaux de relations entretenues avec toutes sortes de partenaires, car, avec les pratiques croissantes d'externalisation, l'entreprise élabore un véritable "espace de transactions" au sein duquel la frontière de l'entreprise devient floue ».

Finalement, la base pyramidale variera donc proportionnellement avec l'importance de la volonté du dirigeant et de la densité de ses relations à l'environnement. Dès lors, la hauteur de la pyramide, comparable à l'intensité de la volonté du dirigeant, permet à l'entreprise de s'orienter plus ou moins durablement sur la voie du succès dans un environnement externe négocié ou subi. Une piste de recherche intéressante pourrait consister à confirmer ou non l'hypothèse selon laquelle il existe une relation entre le succès de la PME et la présence d'un projet fort chez le dirigeant; ce projet pouvant s'exprimer à travers une volonté qui se traduirait par la cohérence des trois pôles : activités, organisation et RH.

\subsubsection{Esquisse d'une GRH en PME}

Comme cela a été décrit précédemment, le dirigeant au poste de commande réalise un ensemble d'activités de gestion, d'administration et d'exécution. À moins d'être seul, il découvre rapidement l'ampleur des questions de GRH, car il devra recruter, sélectionner et intégrer les bonnes personnes et faire partager sa vision du futur. Le dirigeant devra mettre en place un système de rémunération et d'incitation ou 
d'intéressement, clarifier les responsabilités, gérer les relations interpersonnelles, évaluer et améliorer les compétences.

Mais pour les dirigeants de PME, le fait de saisir l'importance de la variable RH dans leur gestion courante et pour leur stratégie ne semble pas constituer un mobile suffisamment fort pour y consacrer le temps, l'attention et les efforts que cette prise de conscience supposerait : il existe un hiatus fort entre leur perception des RH dans le cadre de leur vision stratégique et leurs pratiques de GRH (Deshpende et Golhar, 1994). Au cours d'un précédent travail, l'hypothèse selon laquelle l'importance de ce hiatus pouvait notamment s'expliquer en fonction du profil du dirigeant avait été avancée (Bayad et Nebenhaus, 1993). En effet, la difficulté à mettre en place un programme de GRH provient souvent du style de management du dirigeant qui a pour habitude de traiter les problèmes de RH en face à face et au coup par coup. Faire apparaitre la fonction RH équivaudrait dans son esprit à instiller un élément de bureaucratie incompatible avec le fonctionnement en temps réel de la PME et aussi à devoir clarifier la répartition des activités de chacun au sein de l'organisation (ce qui va à l'encontre du besoin de centralisation du dirigeant).

Pourtant les choix en matière de GRH ont des implications managériales et financières considérables. Ils influencent le développement de l'organisation et du personnel. Face aux incertitudes de l'environnement, les choix appropriés de GRH permettent d'assurer la flexibilité de la PME tant par le développement des compétences que par la capacité de mobilisation et d'intégration des personnes de talent. Finalement pour le dirigeant, un programme de GRH serait un moyen d'action efficace pour répondre de manière cohérente aux défis suivants :

- trouver les meilleures personnes pour les emplois clés par des politiques ouvertes de recrutement et d'intégration des ressources humaines;

- préparer les personnes et les emplois au changement par un système d'appréciation des compétences, par la formation et par le développement de la polyvalence;

- maintenir les performances de la PME par une évaluation des performances individuelles, une bonne définition des emplois, un système réfléchi d'incitation et d'intéressement et une politique de communication active au sein de l'entreprise.

À la lumière du modèle de base de la PME exposé par cet article, le dirigeant peut se rendre compte de l'influence de la GRH dans le système de gestion et de pilotage de son entreprise. Les possibilités d'intervention dans le domaine de la GRH ayant des répercussions sur les variables clés de la compétitivité de la PME peuvent être schématisées comme suit: 


\section{ENVIRONNEMENT}

Marché du travail

Conventions

collectives

Syndicats

États et collectivités

Concurrence

Technologie...

Recrutement

Sélection

Intégration

Formation

Promotion

$\begin{aligned} & \text { Analyse des emplois } \\ & \text { Mémunération } \\ & \text { Motivation } \\ & \text { Compétences }\end{aligned}$
Activités

Par leurs possibilités d'action au regard de chaque variable clé, les pratiques de GRH autorisent un degré de cohérence élevé de la PME. Elles assurent les ajustements nécessaires entre les personnes, les activités et le contexte organisationnel. Elles ouvrent ainsi au dirigeant de PME une voie de réflexion stratégique sur les adéquations suivantes :

- GRH-Activités,

- GRH-Contexte organisationnel,

- Activités-Contexte organisationnel.

Ces mises en cohérence se feront selon une ligne de buts et d'objectifs qui émergera de la mission, de la vision stratégique et de la philosophie de gestion du dirigeant de PME.

\section{Conclusion}

Il va de soi que l'ensemble de ce modèle repose sur l'hypothèse forte d'une autonomie des processus décisionnels de la $\mathrm{PME}$ en dehors de toute dépendance à l'égard d'une GE. Qu'en sera-t-il de l'avenir des PME qui, tout en restant indépendantes et théoriquement conformes au principe précédent, se verront, dans leurs contrats, obligées de se soumettre à des normes extrêmement contraignantes sur le plan de la gestion, de l'organisation et en particulier de la GRH ? Il est fait référence ici à toutes ces PME qui, pour conserver leurs marchés de sous-traitance, sont conduites à appliquer les principes issus des normes ISO 9000 ou aux contraintes posées par l'organisation de la production en flux tendus. 


\section{Bibliographie}

AGEFOS-PME (1992), «Besoins des PME en conseil pour la GRH et la formation », Communication présentée à la conférence nationale.

AMBAO-RAO, S.C. et D. PENDSE (1985), « Human resources compensation and maintenance practices », American Journal Small Business.

BACHELET, C. (1994), «Les pratiques d'information et de communication interne dans les PME », Document de travail, non publié, IUT d'Annecy.

BAYAD, M. (1992), «La gestion des effectifs dans les PMI», Communication présentée au II ${ }^{\mathrm{e}}$ Congrès de l'AGRH, Cergy.

BAYAD, M. (1993), «Internationalisation de la PME et pratiques de GRH », Communication présentée au III ${ }^{\mathrm{e}}$ Congrès de l'AGRH, Lille.

BAYAD, M. et J.L. HERMANN (1991), Les difficultés de la recherche en gestion en milieu $P M E$, ICN-Recherche, Université Nancy II.

BAYAD, M. et D. NEBENHAUS (1993), «Les préoccupations de GRH des dirigeants de PME et leur profil », Communication présentée au IV ${ }^{e}$ Congrès de l'AGRH, Jouy-en-Josas.

Belletante, B. (1991), « Pour une approche des spécificités financières de la PME au travers du concept de territoire financier », RIPME, vol. $4, \mathrm{n}^{\circ} 1$.

Belletante, B. et M. CAlvi (1994), «Méthodologie d'analyse financière des sociétés de service à production géographiquement limitée », Communication présentée à la $39^{\mathrm{e}}$ Conférence annuelle mondiale, ICSB, Strasbourg

BIALES, C. et G. Rolland (1989), «Les PME : atouts et handicaps », Ecoflash, juin.

BRECHET, J.P. (1990), « Pour une analyse stratégique adaptée à la PMI», Revue française de gestion, été 1990.

BODEAU, V. (1994), «Le cadre de référence du dirigeant. Une clé pour la compréhension des stratégies des $\mathrm{PMO} »$, Communication présentée à la $39^{\mathrm{e}}$ Conférence annuelle mondiale, ICSB, Strasbourg.

BRoCKAUS, R.H. (1982), «The psychology of the entrepreneur», dans C.A. Kent et al. (éd.), Encyclopedia of Entrepreneurship, Englewood Cliff, N.J., Prentice Hall.

Cliquet, G. (1992), «Pratiques de recrutement dans les petites entreprises », Cahier de Gestion, $\mathrm{n}^{\circ} 27$, Université de Caen.

COSSETTE, P. (1993), «La vision stratégique du propriétaire-dirigeant de PME », Congrès international francophone de la PME, Carthage, Tunisie.

CossetTE, P. (1994), «Développement d'une méthode systématique d'aide à la mise au point de la vision stratégique chez le propriétaire-dirigeant de $\mathrm{PME} »$, Communication présentée à la $39^{\mathrm{e}}$ Conférence annuelle mondiale, ICSB, Strasbourg.

D'AmBoise, G., Y. GASSE et M. BERNARD (1984), Difficultés managériales et facteurs de succès perçus par les propriétaires-dirigeants de PME, Universités Laval (Québec) et de Nantes (France).

D'AMBoIse, G. (1982), Peut-on faire de la recherche sur la PME ? TIMS/ORSA, Détroit (traduction française de l'Université Laval).

D’AmBoise, G. (1983), «La recherche sur les PME», Options politiques, vol. 4, nº 3. 
D'Amboise, G. et S. Bouchard (1990), « De l'utilisation du concept de vision dans l'étude des organisations », Document de travail 90-02, Université Laval.

D'Amboise, G. et D.J. GARAND (1993), Identification des difficultés et besoins des PME en matière de GRH, Montréal, SDQM.

D'AMBOISE, G. et A. PARENT (1991), «Les problèmes des petites entreprises : ce que révèlent les études nord-américaines », dans Petite entreprise et développement local, Paris, Éditions Eska.

DESHPENDE, S.P. et D.Y. GOLHAR (1994), «HRM practices in large and small manufacturing firms : a comparative study », Journal of Small Business Management, vol. 32, $\mathrm{n}^{\circ} 2$, p. 49-56.

FILION, L.J. (1991), Vision et relations : clefs du succès de l'entrepreneur, Montréal, Édition de l'Entrepreneur, $271 \mathrm{p}$.

FILION, L.J. (1994), «Les systèmes de gestion des propriétaires-dirigeants, entrepreneurs et opérateurs de PME regardés à partir de la métaphore mécanique », Communication présentée à la $39^{\circ}$ Conférence annuelle mondiale, ICSB, Strasbourg.

GARAND, D.J. et B. FABI (1992), «Les pratiques de GRH en PME», Organisation, vol. $2, \mathrm{n}^{\circ} 1$.

GARAND, D.J. et B. FABI (1993), «Les pratiques de conservation des ressources humaines en PME », Communication présentée au IV ${ }^{\mathrm{e}}$ Congrès de l'AGRH, Jouy-en-Josas.

GARTNER, W.B. (1989), «Who is an entrepreneur ? Is the wrong question », Entrepreneurship Theory and Practice, vol. 8, n ${ }^{\circ} 3$, p. 47-68.

GARTNER, W.B. (1990), «What are we talking about when we talk about entrepreneurship? », Journal of Business Venturing, vol. 5, $\mathrm{n}^{\circ}$ 1, p. 15-29.

GASSE, Y. (1983), « Innovation and management as strategies for growth and productivity in small and medium firms », $3^{\mathrm{e}}$ Conférence annuelle de la Strategic Management Society, Paris.

GAUDIN, J. (1990), «L'évolution de l'environnement des PME et son impact sur la GRH», Séminaire IRIS, La GRH dans les PME, Paris, Dauphine.

HORNSBY, J.S. et D.F. KURATKO (1990), «HRM in small business : critical issues for the 1990's », Journal of Small Business Management, vol. 28, nº 3.

JohANNISSON, B. (1985), «Anarchists and organisers », EGCS, $7^{\circledR}$ Colloque, Stockholm.

JOHANNISSON, B. (1994), «Building a "Glocal" strategy, Internationalizing small firms through local networking », Communication présentée à la $39^{\circ}$ Conférence annuelle mondiale, ICSB, Strasbourg.

JULIEN, P.A. (1993), « Globalisation de l'économie et PME », Communication présentée au Congrès international francophone de la PME, Carthage, Tunisie.

JULIEN, P.A. (1993), Les PME : technologie et compétitivité, OCDE.

JULIEN, P.A. et M. MARCHESNAY (1988), La petite entreprise : principes d'économie et de gestion, Paris, Vuibert-Gestion, 288 p.

KAO, J. (1991), « The Entrepreneurial Organization », Englewood Cliff, N.J., Prentice Hall.

LARRIVÉE, L. et G. D'AMBOISE (1989), « Difficultés de recrutement dans les PME québécoises », Relations industrielles, vol. 44, $\mathrm{n}^{0} 3$. 
LENY, O.M. (1992), «Impact de l'environnement externe et interne sur la définition des politiques de GRH dans les PME », Communication présentée au $\mathrm{III}^{\mathrm{e}}$ Congrès de l'AGRH, Lille.

LiouviLle, J. et M. BAYAD (1994), «Stratégies de gestion des RH et performances dans les $\mathrm{PME} »$, Communication présentée à la $39^{\circ}$ Conférence annuelle mondiale, ICSB, Strasbourg.

LORRAIN, J., A. BELley et C. RAMANGALAHY (1994), « Relation entre le profil de comportement des propriétaires-dirigeants et le stade d'évolution de leur entreprise », Revue internationale PME, vol. 7, $\mathrm{n}^{\circ}$ 1, p. 9-34.

LORRAIN, J. et L. DUSSAUlT (1988), «Les entrepreneurs artisans et opportunistes : une comparaison de leurs comportements de gestion », Revue internationale PME, vol. 1 , $\mathrm{n}^{\mathrm{o}} 3$, p. 157-176.

MCEvoy, G.M. (1983), «Personnel practices in smaller firms », American Journal of Small Business, vol. $8, \mathrm{n}^{\circ} 2$.

MARCHESNAY, M. (1991), «La PME : une gestion spécifique ?», Économie rurale, $\mathrm{n}^{\circ} 206$.

MARCHESNAY, M. (1997), «Le management stratégique », dans GREPME, Les PME : bilan et perspectives, Ouvrage collectif, $2^{\mathrm{e}}$ édition, Cap-Rouge, Presses Inter Universitaires, Paris, Economica.

MinTZBERG, H. et J. Waters, (1985), «Of strategies, deliberate and emergent », Strategic Management Journal, vol. 6.

MONNOYER-LONGE, M.C. (1994), «Construction résiliaire et développement des PME de service aux entreprises », Communication présentée à la $39^{\mathrm{e}}$ Conférence annuelle mondiale, ICSB, Strasbourg.

NeBENHAUS, D. (1990), «Les structures participatives d'influence du personnel sur les décisions », RIPME, vol. 3, $\mathrm{n}^{\circ} 1$.

NEBENHAUS, D. et P. SARNIN (1993), «Problématiques et spécificités des recherches de GRH dans les PME », Communication présentée au IV ${ }^{e}$ Congrès de l'AGRH, Jouy-en-Josas.

PACHE, G. (1991), «L'impact des stratégies d'entreprises sur l'organisation industrielle: PME et réseaux de compétences », Revue d'Économie industrielle, vol. 56, $\mathrm{n}^{\circ} 2$.

PARADAS, A. (1992), «L'évaluation des résultats de la formation professionnelle dans les PME », Communication présentée au III $^{\mathrm{e}}$ Congrès de l'AGRH, Lille.

PARADAS, A. (1993), «Les conditions de succès d'une politique de formation en PME », Communication présentée au Congrès international francophone de la PME, Carthage, Tunisie.

PIORE, M.J. et C.F. SABEL (1984), The Second Industrial Divide, New York, Basics Books.

ROCHA, J.R. et M. RIAZ KHAN (1985), «The human resource factor in small business decision making », American Journal of Small Business.

SARNIN, P. (1992), «La GPE dans les PME du quantitatif au qualitatif », Communication présentée au III' Congrès de l'AGRH, Lille.

SHAPERO, A. et L. Sokol (1982), «The social dimensions of the entrepreneurship », dans C.A. Kent et al. (éd.), Encyclopedia of Entrepreneurship, Englewood Cliff, N.J., Prentice Hall.

VeSPER, K.H. (1980), « New venture strategies », Englewood Cliff, N.J., Prentice Hall. 